\title{
Technique of endovascular repair of iatrogenic subclavian artery injury following subclavian vein catheterization
}

\author{
Marek Kazibudzki', Jerzy Wojciech Krzywoń', Tomasz Kwiatkowski', \\ Katarzyna Zbierska- Rubinkiewicz', Elżbieta Dobrowolska², Mariusz Trystuła' \\ 'Department of Vascular Surgery, John Paul II Hospital, Krakow, Poland \\ ${ }^{2}$ Department of Anesthesiology and Intensive Care, John Paul II Hospital, Krakow, Poland
}

\begin{abstract}
The iatrogenic subclavian artery injury is a rare but potentially serious complication of subclavian vein cannulation. The use of endovascular techniques is an alternative to surgical repair especially in patients with concomitant diseases in whom immediate, potentially large surgery would be associated with a high risk of perioperative complications. This article discusses technical aspects of endovascular repair of iatrogenic injury of subclavian artery with implantation of covered stents based on two cases. Author's experience and data from literature suggests that endovascular management including covered stent implantation is safe and effective treatment and should be considered as a method of choice especially among patients in poor general condition and/or major comorbidities.
\end{abstract}

Key words: subclavian artery, covered stents, central catheters, iatrogenic injury

Acta Angiol 2020; 26, 4: I50-154

\section{Introduction}

Central venous catheter (CVC) insertion is a frequently performed procedure. The most commonly cannulated vessels are the internal jugular, subclavian and femoral vein. The iatrogenic subclavian artery injury described in this paper is a rare but potentially serious complication of large vein catheterization, especially when large diameter dialysis catheters are used. The outflow of arterial blood from the cannula is the evidence of an incorrect position of the catheter in the artery. X-ray, ultrasound and Angio CT are helpful in confirming the diagnosis. Considering relatively difficult surgical approach to subclavian artery and frequently poor general condition of patients undergoing central veins cannulation, endovascular repair of injured artery seems reasonable.

\section{Cases report}

The paper presents two cases (a 62-year-old male and a 44-year-old female) of iatrogenic injury of the subclavian artery following the attempt of subclavian vein cannulation with a central venous catheter. In both cases, the catheter was misplaced in the subclavian artery. Artery injury did not cause a significant deterioration of patient's clinical condition. However, due to the underlying diseases and comorbidities, it was necessary to choose the least burdensome method of catheter removal and local repair of the vessels,

Address for correspondence: Tomasz Kwiatkowski, Department of Vascular Surgery, John Paul II Hospital, Krakow, Poland, Prądnicka 80, 3I-202 Kraków, Poland; e-mail: tomkwiatkowski@go2.pl 

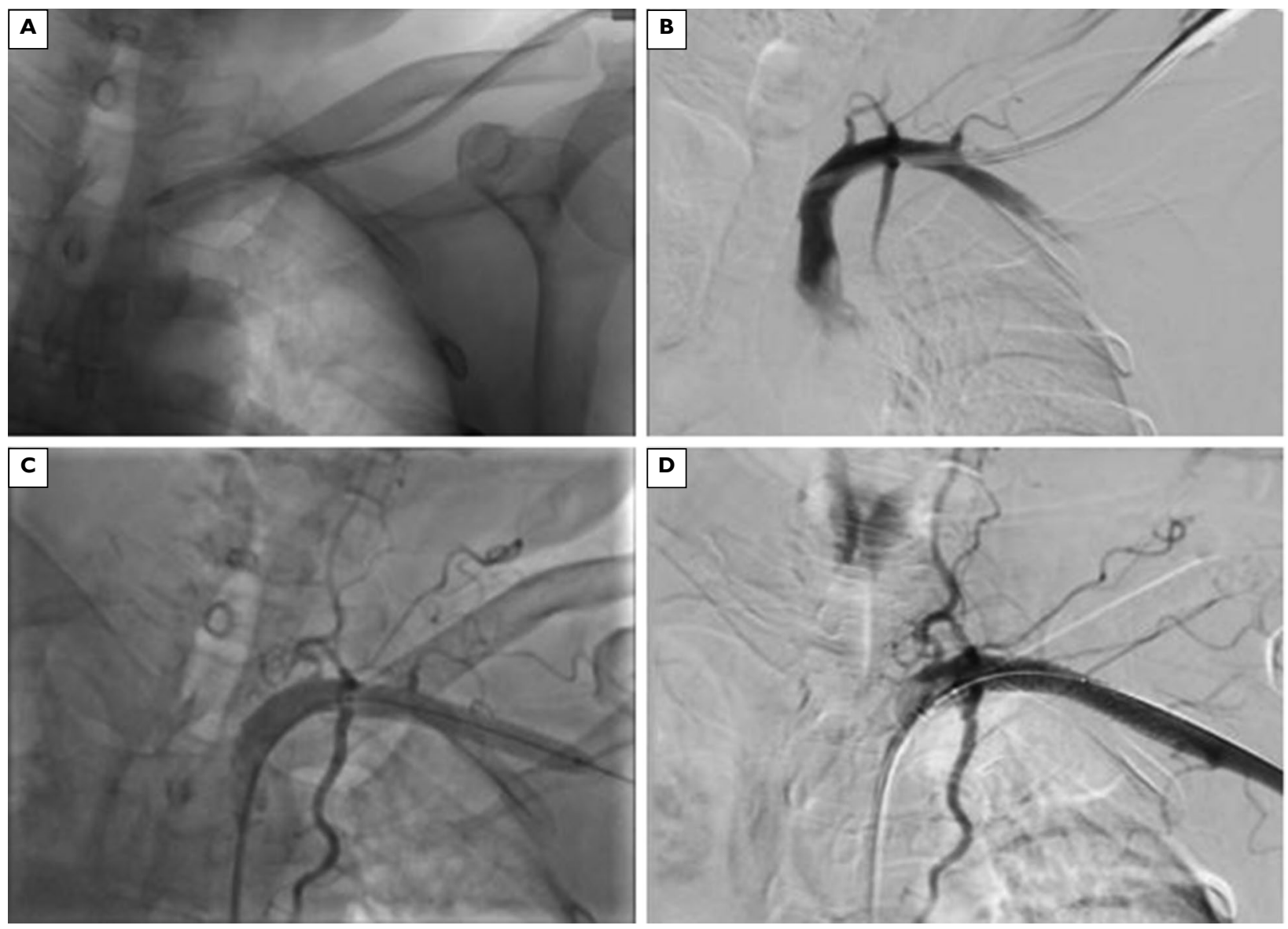

Figure I. Case I: A — location of the catheter using angiography; B — subclavian artery with a catheter; C — stent positioning; $D$ - after stent implantation

preferably avoiding open surgery. In both cases, the procedure consisted of catheter removal under fluoroscopic control and endovascular repair of the damaged artery with a covered stent. Another possible method of treatment would be hybrid operation with use of balloon for temporary occlusion of the vessel and surgical repair or open vascular repair, a very complicated and risky procedure in both patients.

\section{Surgical technique}

Local anesthesia with I\% lignocaine solution was used at the CVC puncture site and access site to the common femoral artery in both cases and additionally at the right brachial artery in the second patient. In both cases puncture and insertion of the 7F sheath into the common femoral artery (CFA) was performed under ultrasound guidance. Next, guidewire supported with diagnostic catheter was introduced through injured subclavian artery (SA) to peripheral part of brachial artery. Subsequently, the previously used short sheath was replaced with a long one, introduced to the prox- imal part of the SA. Injury of the vessel was precisely localized in fluoroscopy and diameter of artery was measured to select appropriate balloon expandable covered stent.

In both cases an additional leakage test was performed using the balloon catheter with a nominal diameter I mm larger than the diameter of the vessel at the site of injury. Absence of both contrast flow to the peripheral part of the artery and it's extravasation after simultaneous central catheter removal and balloon inflation in the injury site confirmed appropriate choice of covered stent diameter. In the first case stent was introduced via femoral access. The same way was used for angiography. In the second case (due to different vessels anatomy) stent was introduced through brachial access, and angiographic control was performed with femoral access for better landing precision. CVC removal and implantation of a covered stent under fluoroscopic control were performed simultaneously to minimize bleeding from artery perforation. Angiographic control of the stent tightness revealed no leakage. In the second case, it was necessary to cover the ostium 

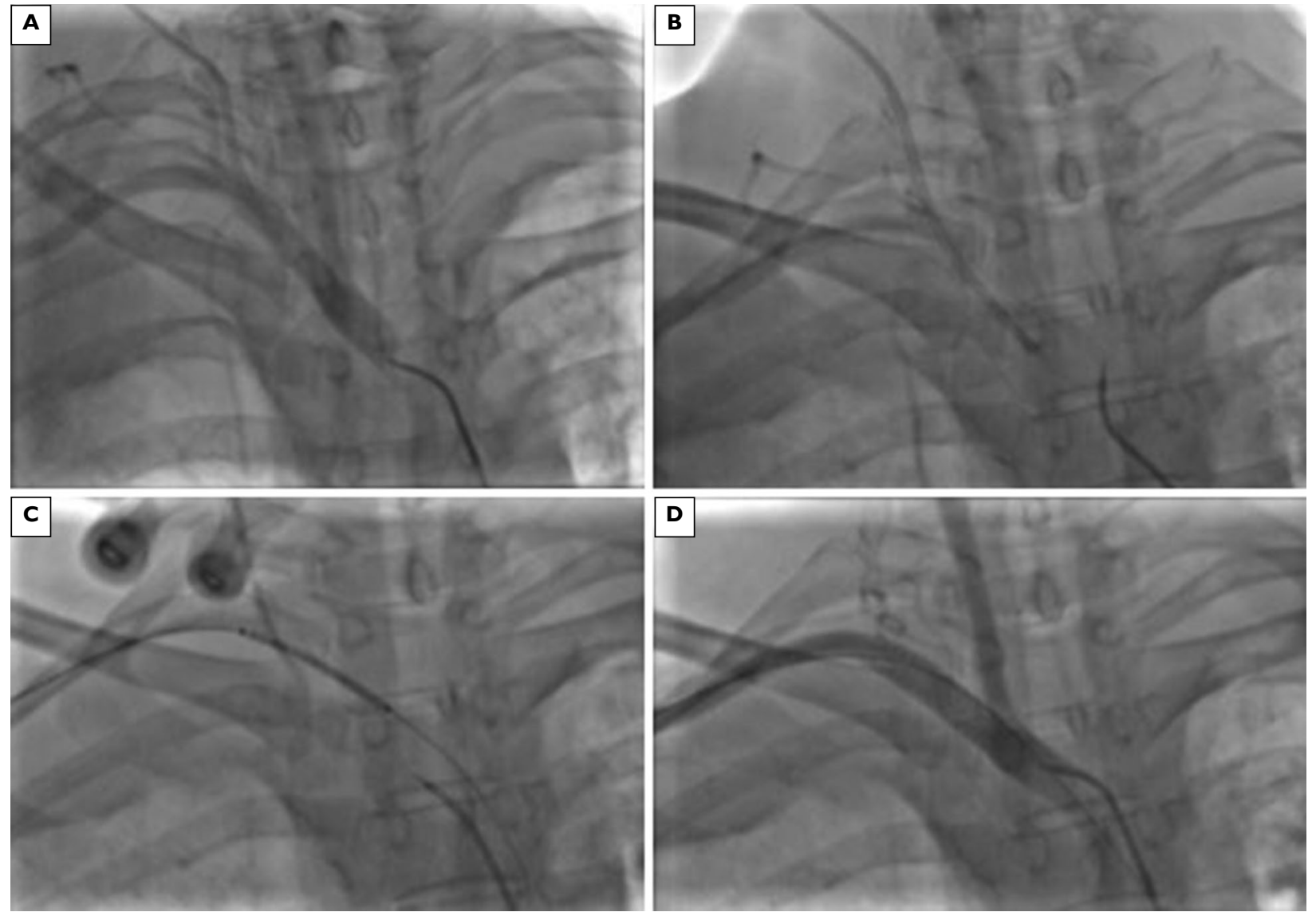

Figure 2. Case 2: A, B — location of the catheter in the vessel; C — stent positioning; D — after stent implantation

of the right vertebral artery (VA), due to its proximity of the puncture site. No neurological deficits associated with the VA coverage were observed. In both cases, the access site to CFA was closed using AngioSeal system. Both patients were discharged to their primary hospitals with no signs of leakage (bleeding) or hemoglobin level decrease.

Procedure - step by step:

I. Ultrasound-guided puncture of CFA and introduction of short sheath 7F.

2. Introduction of hydrophilic guide wire 0.035 " (Terumo) and diagnostic catheter Bern 4F (Boston Scientific) to the injured SA. Exchange of wire for the Supra Core 35 Guide Wire (Abbott).

3. Exchange of the short sheath $7 F$ for the long $7 F$ Destination (Terumo) (with a diameter guaranteeing contrast application when positioning the stent in the case of using this sheath as a working channel as in case I) or performing access to brachial artery for retrograde stent delivery as in case 2 . In the second case, diagnostic catheter previously introduced via femoral access was used for angiographic control during positioning and implantation of the stent.
4. Measurement of the vessel diameter and the use of a balloon catheter (Armada 35, Abbott) inflated in the site of future stent implantation to confirm proper stent diameter.

5. Simultaneous removal of a foreign body and expansion of the PTFE covered stent Advanta VI2 (Atrium) on the balloon to minimize bleeding from the site of damage.

6. Angiographic control, removal of the catheter and sheath, access site closure with Angio-Seal.

\section{Discussion}

Central access is obtained using anatomical landmarks or with ultrasound/ fluoroscopy guidance. The first technique is characterized by a high percentage of failures and complications of $30 \%$ and $18.8 \%$, respectively [I]. The use of ultrasound significantly reduced the number of failures, and attempts needed to gain central vein access as well as the frequency of complications such as accidental puncture of the artery, hematoma, pneumothorax, etc. Ultrasound-guided access to the subclavian vein is more difficult (compared to the 
internal jugular and femoral vein) due to anatomical conditions. Consequently, the risk of complications is higher in this access site, especially because the subclavian artery, due to its anatomical location, cannot be effectively compressed and the catheters used have a significantly higher diameter [2]. Therefore, the injury to the subclavian artery by erroneous puncture may result in pseudoaneurysm, arteriovenous fistula or uncontrolled bleeding, especially that puncture attempts are often performed several times [3-5]. It seems that insertion into the jugular vein under ultrasound control is a more reliable and safer alternative to subclavian access, although damage at this level is more frequently reported. Mortality of up to $30 \%$ was reported as a result of damage of SA and proximal part of the axillary artery [6]. For this reason as well as due to technical difficulties of open surgery, techniques of endovascular repair of these vessels injuries are currently preferred. They can be used separately or as part of a hybrid method involving prior endovascular bleeding control using balloon catheter followed by open surgical repair.

If the catheter is accidentally positioned in the artery, the best way of action is to secure it with dressing and contact the vascular center. Because in most cases the catheter alone allows to maintain local hemostasis, it gives time to prepare for surgery. The use of endovascular techniques, such as covered stent implantation, temporary balloon closure, embolization, and the use of thrombin, is an alternative to surgical repair, especially in patients with concomitant diseases in whom immediate, potentially large surgery would be associated with a high risk of perioperative complications. Due to large diameter of implanted catheters in our patients, the use of closure device was expected to be inefficient. In these cases endovascular repair with precise damage control seems to be optimal choice. In cases in which catheter was already removed with subsequent pseudoaneurysm, bare metal stent with additional embolization can be considered. If endovascular repair fails and active bleeding is still present, open surgical repair is inevitable, but was never necessary in our experience.

The open surgical reconstruction is the treatment of choice for extensive vascular injury, active arterial bleeding and pleural damage $[7,8]$. In our opinion in other cases, the use of a covered stent, if possible, should be the treatment of choice. Generally in case of SA lesion, self-expandable stents are preferred especially in case of variable vessel diameter ant it's tortuosity. In these particular cases of artery injury, balloon expandable stents were used due to the possibility of precise implantation, avoidance of additional maneuvers (stent postdilatation) connected with potential blood loss. Furthermore, both patients were in severe medical condition (with short life expectancy); thus, risk of future stent damage caused by limb movements (mechanism of the clavicle-ribbed scissors) was considered acceptable. It is important to accurately verify the size, both diameter and length of a stent, to avoid leakage and necessity of implantation of additional stents [9]. In the technique described above, it was helpful to pre-seal the vessel with a balloon catheter of diameter $1 \mathrm{~mm}$ greater than the vessel measured under fluoroscopy and length equal to pre-selected stent in order to confirm proper stent selection. It is important to use a single stent with a length and diameter that guarantees hemostasis. Simultaneous expansion of the stent and removal of a foreign body (CVC) under fluoroscopic control minimizes the possibility of uncontrolled blood loss. In the case of difficult anatomy or proximity of the puncture site to the ostium of the vertebral artery (VA) like in the second case or other important anatomical structure, additional access from the brachial artery should be considered to optimize visualization of stent deployment.

\section{Conclusions}

latrogenic injury of the subclavian artery following an attempt of subclavian vein cannulation can be effectively and safely treated with endovascular techniques. In our opinion, endovascular management should be considered as a method of choice especially among patients in poor general condition and/or with major comorbidities. The key points for successful procedure are precise localization of artery injury site with high quality angiogram and proper selection of covered stent length and diameter. Depending on vessels anatomy and injury localization, simultaneous use of both femoral and brachial accesses may be required. In case of extensive vascular injury, when open surgery may be inevitable, prior endovascular bleeding control using balloon catheter is strongly recommended.

\section{Conflict of interest}

None.

\section{References}

I. Sznajder JI, Zveibil FR, Bitterman H, et al. Central vein catheterization. Failure and complication rates by three percutaneous approaches. Arch Intern Med. 1986; I46(2): 259-26I, doi: 10.100 I/archinte.146.2.259, indexed in Pubmed: 3947185.

2. Rossi UG, Petrocelli F, Ferro C. Subclavian artery pseudoaneurysm complicating central venous catheterization: endovascular treatment with Amplatzer Vascular Plug 4 and covered stent. Catheter Cardiovasc Interv. 2013; 82(7): E906-E910, doi: 10.1002/ccd.24796, indexed in Pubmed: 23359531.

3. Wheeler SC, Zinn KM, Hughes TW. Endovascular covered stent repair of an iatrogenic subclavian artery-to-pulmonary artery 
fistula and pseudoaneurysm. J Vasc Interv Radiol. 2007; 18(6): 775-779, doi: 10.1016/j.jvir.2007.02.029, indexed in Pubmed: |7538|4|.

4. Stoica SC, Fleet M, Howd A. Subclavian artery injury following percutaneous insertion of dialysis catheter. Rev Med Chir Soc Med Nat lasi. 1998; 102(3-4): 194-197, indexed in Pubmed: 10756874.

5. Cox CS, Allen GS, Fischer RP, et al. Blunt versus penetrating subclavian artery injury: presentation, injury pattern, and outcome. J Trauma. 1999; 46(3): 445-449, doi: 10.1097/00005373199903000-00017, indexed in Pubmed: 10088848.

6. Waller CJ, Cogbill TH, Kallies KJ, et al. Contemporary management of subclavian and axillary artery injuries - A Western Trau- ma Association multicenter review. J Trauma Acute Care Surg. 2017; 83(6): 1023-1031, doi: 10.1097/TA.0000000000001645, indexed in Pubmed: 287I5360.

7. Tawfic QA, Bhakta P, Mohammed AK, et al. Subclavian vein injury and massive hemothorax requiring thoracotomy following insertion of tunneled dialysis catheter--a case report and review of literature. Middle East J Anaesthesiol. 2010; 20(6): 86I-864, indexed in Pubmed: 21526674.

8. Maciejewski DR, Tekieli $\measuredangle$, Machnik R, et al. Simultaneous vertebral and subclavian artery stenting. Postepy Kardiol Interwencyjnej. 2017; 13(2): 142-149, doi: 10.5। |4/pwki.2017.68052, indexed in Pubmed: 28798785. 